\title{
Atresia of small intestine
}

INSERM

\section{Source}

INSERM. (1999). Orphanet: an online rare disease and orphan drug data base. Atresia of small intestine. ORPHA:1201

Atresia of small intestine is a special form of intestinal atresia with absence of mesentery, which is most likely due to an intrauterine intestinal vascular accident. Newborns are usually preterm infants with low birth-weights, that encounter feeding difficulties (including vomiting with initial feeds, which may later worsened and the abdomen becomes progressively distended) as well as failure to thrive. Affected children present disrupted bowel loops assuming a spiral configuration resembling an 'apple peel' and may have less than half of the normal length of the small bowel and a physiologically short bowel. Atresia of small intestine is characterized by jejunal atresia near the ligament of Treitz, foreshortened bowel, and a large mesenteric gap. The bowel distal to the atresia is precariously supplied. Atresia of small intestine may be a manifestation of cystic fibrosis (see this term). The most important cause of mortality is short bowel syndrome (see this term), encountered in $65 \%$ of cases. 